\title{
Cell Adhesion and Proliferation onto Chitosan-based Membranes Treated by Plasma Surface Modification
}

\author{
Sandra M. Luna, Simone S. Silva, ${ }^{*}$ Manuela E. Gomes, \\ JOÃO F. MANO AND RUI L. REIS \\ 3B's Research Group - Biomaterials, Biodegradables \\ and Biomimetics, Department of Polymer Engineering \\ University of Minho, Headquarters of the European Institute of Excellence \\ on Tissue Engineering and Regenerative Medicine - AvePark, Zona \\ Industrial da Gandra - Caldas das Taipas 4806-909, Guimarães, Portugal \\ IBB-Institute for Biotechnology and Bioengineering \\ PT Government Associated Laboratory, Guimarães, Portugal
}

\begin{abstract}
Surface properties play a vital role in the functioning of a biomaterial. Cellular adherence and growth onto biomaterials can be enhanced in biomaterial modifications of their surface. In this work, the cell behavior on chitosan membranes modified by argon and nitrogen-plasma treatments was investigated. Characterization of the membranes was performed using atomic force microscopy, contact angle measurements, and X-ray photoelectron spectroscopy. Cytotoxicity assessment and direct contact assay were carried out for untreated and treated chitosan membranes using L929 fibroblast-like cells. Cell morphology and cell viability were assessed to evaluate the cell attachment and proliferation. Changes in terms of roughness, surface chemistry, and hydrophilicity/hydrophobic balance of chitosan-modified membranes were observed. Regarding cell studies, the findings revealed that the extracts of all membranes do not induce cytotoxic effects. Moreover, the in vitro assays evidenced an improvement of the L929 adhesion and attachment when compared to untreated chitosan membranes. Overall, the data obtained clearly demonstrated that plasma
\end{abstract}

*Author to whom correspondence should be addressed.

E-mail: simonesilva@dep.uminho.pt

Figures 2 and 3 appear in color online: http://jba.sagepub.com

JOURNAL OF BIOMATERIALS APPLICATIONS Vol. 26 - July 2011 
treatments constitute an effective way of improving the biocompatibility of chitosan membranes towards to their use in biomedical applications.

KEY WORDS: chitosan membrane, plasma treatment, cell behavior, surface properties, biomaterial.

\section{INTRODUCTION}

$\mathbf{N}$ owadays, much attention has been paid to surface modification of biomaterials as a way to enhance both their performance and biocompatibility. Many methods are employed for modifying polymer surfaces including grafting [1], gamma irradiation [2], and plasma surface modification [3-8]. Among them, plasma treatment is a method widely used to modify the surface functionality. Depending on the used conditions (atmosphere, exposure time, and power), a surface can be modified to become hydrophilic or hydrophobic or only be activated to further reactions [7].

Chitosan is a known natural polymer obtained by alkaline deacetylation of chitin $[9,10]$. This polymer has interesting properties including bioadhesiveness, biodegradability, and bacteriostatic activities. Chitosan-based matrices such as gels, porous structures, fibers, membranes, and capsules have been widely investigated for several biomedical applications such as wound healing [11-15], drug delivery [16], cell encapsulation [17], orthopedic/periodontal applications [10], and tissue engineering [10,18]. Previous studies [19] report that chitosan has intrinsic accelerating wound healing properties. The most important characteristics of chitosan for its use as wound dressing are related to hemostatic action, biodegradability, biocompatibility, retention of growth factors, release of glucosamine and $\mathrm{N}$-acetyl glucosamine monomers and oligomers, and stimulation of cellular activities [16]. Studies [19] suggest that chitin and chitosan stimulate the migration of polymorphonuclear and mononuclear cells, and accelerate the reformation of connective tissue and angiogenesis.

Previous study performed by our group [4] has shown that surface properties such as roughness, wettability, and surface chemistry on chitosan membranes were clearly modified through the plasma surface treatment using nitrogen and argon plasma. These changes on the membrane surface influenced their cell behavior with respect to nonmodified chitosan membrane. In this present study, we have investigated, in more detail, the biological response of fibroblasts seeded onto these plasma-treated chitosan membranes. 


\section{MATERIALS AND METHODS}

\section{Materials}

Chitosan-cht (Sigma Adrich) with deacetylation degree of $83.8 \%$ and molecular viscosimetry weight of $662 \mathrm{kDa}$ was used. All other reagents were analytical grade and used as received. Chitosan membranes (average thickness of $47 \mu \mathrm{m}$ ) were prepared by solvent casting, neutralized with sodium hydroxide $(\mathrm{NaOH})$, and subsequently the dried membranes were submitted to plasma treatment, as described previously [4]. Briefly, the plasma treatment was carried out in a radio frequency plasma reactor (PlasmaPrep5, Germany). The plasma chamber was thoroughly purged with a continuous flow of the gas used during the treatment to reduce trace amounts of air and moisture. A power of $20 \mathrm{~W}$ was applied. The duration of the glow discharge treatment was varied from 10 to $40 \mathrm{~min}$. The membranes treated with nitrogen plasma were designated as ChtP1-ChtP4 while the treated with argon plasma were identified as ChtP5-ChtP8 (Table 1). Untreated chitosan membranes (cht) were used as control.

\section{Characterization Methods}

\section{Atomic Force Microscopy}

The roughness of the sample surface was measured by AFM. The analyses were performed on at least three spots per sample using TappingMode (Veeco, USA) connected to a NanoScope III (Veeco, USA) with noncontacting silicon nanoprobes (ca $300 \mathrm{kHz}$, setpoint 2-3 V) from Nanosensors, Switzerland. All images were fitted to a plane using the third-degree flatten procedure included in the NanoScope software version $4.43 \mathrm{r} 8$. The surface roughness was calculated as Sq (root mean square from average flat surface) and Sa (average absolute distance from average flat surface). The values are presented as mean \pm standard deviation.

Table 1. Plasma conditions used on chitosan membranes.

\begin{tabular}{lcccccccc}
\hline $\begin{array}{l}\text { Conditions/ } \\
\text { Samples }\end{array}$ & ChtP1 & ChtP2 & ChtP3 & ChtP4 & ChtP5 & ChtP6 & ChtP7 & ChtP8 \\
\hline Power (W) & 20 & 20 & 20 & 20 & 20 & 20 & 20 & 20 \\
Time (min) & 10 & 20 & 30 & 40 & 10 & 20 & 30 & 40 \\
Gas & Nitrogen & Nitrogen & Nitrogen & Nitrogen & Argon & Argon & Argon & Argon \\
\hline
\end{tabular}




\section{Contact Angle Measurements}

The surface wettability of the membranes was assessed by static contact angle $(\theta)$ measurements using the sessile drop method. Two different liquids were used: ultra-pure water (polar) and diiodomethane (nonpolar). The measurements were performed using OCA20 equipment (DataPhysics, Germany) and SCA-20 software. The presented data were averaged on six measurements.

\section{X-ray Photoelectron Spectroscopy}

The XPS analysis was performed using ESCALAB 200A, VG Scientific (UK) with PISCES software for data acquisition and analysis. For analysis an achromatic $\mathrm{Al}(\mathrm{K} \alpha)$ X-ray source operating at $15 \mathrm{kV}(300 \mathrm{~W})$ was used. The spectrometer was calibrated with reference to $\mathrm{Ag} 3 \mathrm{~d} 5 / 2$ $(368.27 \mathrm{eV})$ and it was operated in constant analyzer energy (CAE) mode with $20 \mathrm{eV}$ pass energy. The measurements were carried out at a take-off angle of $90^{\circ}$ (normal to the surface). Data acquisition was performed with a pressure lower than $10^{-6} \mathrm{~Pa}$. The value of $285 \mathrm{eV}$ of the hydrocarbon $\mathrm{C} 1 \mathrm{~s}$ core level was used as a calibration for the absolute energy scale. Overlapping peaks were resolved into their individual components by XPSPEAK 4.1 software.

\section{In Vitro Cell Culture Studies}

Prior to cell culture studies, all the membranes (untreated and treated) were sterilized by ethylene oxide.

\section{Cytotoxicity Screening}

In order to assess the eventual cytotoxicity of the treated membranes, extracts of all membranes were prepared and placed in contact with a mouse fibroblast-like cell line, L929 (L929 cells; European Collection of Cell Cultures-ECACC, UK) and tested using a MTT test assay in accordance to protocols described in ISO/EN10993 [20]. For this test, extracts of the membranes were obtained by their immersion in culture medium for $24 \mathrm{~h}$ at $37^{\circ} \mathrm{C}, 60 \mathrm{rpm}$. The filtered extracts were placed in contact with a monolayer of L929 cells for $72 \mathrm{~h}$. Then, the cell viability was evaluated by MTT assay, and the results were expressed as percentage of cell viability.

\section{Cell Culture}

A cell line of mouse lung fibroblast (L929) was selected for all in vitro assays. This cell line is also commonly used for assessment of the cytotoxicity of the new biomaterials as these cells are able to maintain 
their phenotypic characteristics for long periods of culture [21], and therefore these tests can be more reproducible. Cells were cultured in basic medium composed of Dulbecco's Modified Eagle's Medium - low glucose (DMEM; Sigma-Aldrich), supplemented with $10 \%$ fetal bovine serum (FBS; Biochrom AG; Germany), 1\% antibiotic/antimycotic (Invitrogen), and sodium bicarbonate (Sigma), at $37^{\circ} \mathrm{C}$ in a $5 \% \mathrm{CO}_{2}$ until achieving $90 \%$ of confluence [22]. For MTT (3-(4,5-dimethylthiazol-2-yl)-2,5-diphenyltetrazolium bromide - Sigma) assay, a cell suspension was prepared with a concentration of approximately $6.6 \times 10^{4} \mathrm{cell} / \mathrm{mL}$ and seeded onto 96 -well plates. The results were expressed as percentage of cell viability.

\section{Direct Contact Assay - Assessment of Cell Adhesion and Proliferation of L929 Cells Seeded onto Chitosan Membranes}

The Direct Contact assay was used to evaluate the cell adhesion and cell proliferation on the surface of chitosan membranes modified by different argon and nitrogen plasma treatments. L929 fibroblast cells were initially harvested from monolayer cultures using trypsin-EDTA. A cell suspension was prepared in order to obtain a cell density of $8 \times 10^{4}$ cells $/ \mathrm{cm}^{2}$ [23]. The membranes were placed in 24-well plates and $100 \mu \mathrm{L}$ of this cell suspension was seeded onto the samples surface $\left(1 \times 1 \mathrm{~cm}^{2}\right)$. Empty wells and coverslips (Sarstedt, USA) were also seeded with the same amount of the cell suspension to be used as controls. All the seeded samples and controls were incubated at $37^{\circ} \mathrm{C}$ in an atmosphere containing $5 \%$ of $\mathrm{CO}_{2}$ for $2-3 \mathrm{~h}$. After this time, $1.5 \mathrm{~mL}$ of DMEM culture medium was added into each culture well and the samples cultured for different periods of time, namely 3,7 , and 14 days. The culture medium was changed every 2 days.

The cell adhesion and proliferation was assessed qualitatively using light microscopy (after blue methylene staining), scanning electron microscope (SEM), and quantitatively using the MTS test.

\section{SEM Analysis of the Membranes}

Prior to SEM measurements the seeded membranes were fixed with $2.5 \%$ glutaraldehyde (Sigma-Aldrich, Germany) in a PBS solution and then dehydrated using a series of ethanol solutions $(25,30,50,70,80$, $90,100 \%, \mathrm{v} / \mathrm{v})$. The samples were dried overnight at room temperature, coated with gold by sputtering and observed by SEM.

\section{MTS Test - Cellular Viability}

Cellular viability and proliferation were assessed by the MTS-CellTiter $96^{\circledR}$ aqueous one cell proliferation assay (Promega, Madison, USA). 
The MTS (3-(4,5-dimethylthiazol-2-yl)-5-(3-carboxymethoxyphenyl)-2(4-sulfophenyl)-2H-tetrazolium) test has been described previously [24,25]. Briefly, in this test a substrate - the MTS - is bioreduced into a brown formazan product by dehydrogenase enzymes in metabolically active cells $[24,25]$. In this study, MTS solution was prepared with DMEM without phenol red and FBS in a 5:1 ratio, where $300 \mu \mathrm{L}$ of MTS solution was added to membranes/cells and incubated for $3 \mathrm{~h}$ at $37^{\circ} \mathrm{C}$, in an atmosphere containing $5 \%$ of $\mathrm{CO}_{2}$ in the dark. After this incubation period, $100 \mu \mathrm{L}$ of solution from each membrane was transferred to 96-well plates (in triplicate) and the absorbance was determined at $490 \mathrm{~nm}$ using a microplate reader (BIO-TEK - Synergy HT). The results were expressed as absorbance obtained for the samples in each culture period.

\section{Statistical Analysis}

Statistical differences in cell viability of the different samples studied were determined by Student's $t$-test. $p<0.05$ was considered statistically significant.

\section{RESULTS AND DISCUSSION}

In plasma surface modification, two competitive processes can occur, namely functionalization and etching, which will depend of the working conditions [26]. Ongoing etching processes were confirmed by AFM. The roughness values increased after longer exposure time (30 and $40 \mathrm{~min}$ ) when nitrogen was used as a working gas (Table 2). However, shorter exposure time (10 and $20 \mathrm{~min}$ ) was more effective when the modification was performed in an argon plasma atmosphere.

Table 2. Properties of original and modified membranes.

\begin{tabular}{|c|c|c|c|c|c|}
\hline \multirow[b]{2}{*}{ Treatment } & \multirow[b]{2}{*}{ Sample } & \multirow[b]{2}{*}{ Roughness } & \multirow[b]{2}{*}{$\theta_{\text {Water }}\left({ }^{\circ}\right)$} & \multicolumn{2}{|c|}{ Atomic ratio (\%) } \\
\hline & & & & $\mathrm{C} / \mathrm{O}$ ratio & $\mathrm{C} / \mathrm{N}$ ratio \\
\hline \multirow{4}{*}{$\mathrm{N}_{2}$ Plasma } & Chitosan & $2.8 \pm 0.8$ & $88.5 \pm 1.6$ & 2.37 & 11.82 \\
\hline & ChtP1 & $5.6 \pm 1.4$ & $87.7 \pm 1.7$ & 2.12 & 11.24 \\
\hline & ChtP2 & $3.8 \pm 0.3$ & $85.1 \pm 9.4$ & 2.90 & 13.20 \\
\hline & ChtP3 & $7.2 \pm 0.9$ & $83.5 \pm 5.6$ & 3.27 & 8.21 \\
\hline \multirow{5}{*}{ Ar plasma } & ChtP4 & $7.8 \pm 2.1$ & $84.7 \pm 7.1$ & 2.76 & 9.05 \\
\hline & ChtP5 & $3.7 \pm 0.6$ & $78.3 \pm 9.4$ & 2.21 & 7.84 \\
\hline & ChtP6 & $7.3 \pm 0.9$ & $88.1 \pm 3.3$ & 2.73 & 7.61 \\
\hline & ChtP7 & $9.5 \pm 2.2$ & $84.9 \pm 4.4$ & 2.84 & 10.57 \\
\hline & ChtP8 & $5.8 \pm 0.4$ & $93.7 \pm 2.6$ & 2.29 & 11.33 \\
\hline
\end{tabular}


On the other hand, modifications in the morphology and surface chemistry can change the surface hydrophilicity [26], which is one of key surface parameters determining the material/bioenvironment interactions. As it can be seen in Table 1, all the treatments resulted in more hydrophilic surfaces. Furthermore, the XPS results indicated that a decrease in the $\mathrm{C} / \mathrm{N}$ ratio for shorter treatment times (10 and $20 \mathrm{~min}$ ) when argon plasma was used (Table 2). As argon is nonreactive gas, these results can be associated mainly to the etching process. However, when the treatment was extended this relation was not that straightforward. When nitrogen is used as a working gas, not only etching but functionalization with incorporation of $\mathrm{N}$-containing groups $\left(-\mathrm{NH}_{2}\right.$, $\mathrm{NH},=\mathrm{NH}, \mathrm{CONH}_{2}$, or $\mathrm{C} \equiv \mathrm{N}$ ) was expected [27]. We observed that longer exposure times (30 and $40 \mathrm{~min}$ ) resulted in higher nitrogen content (Table 2), which suggested the incorporation of those groups on the material surface.

\section{In Vitro Cytotoxicity Assessment}

Cytotoxicity Effect of Leachables Obtained from Chitosan Membranes Treated by Argon and Nitrogen Plasma on L929 Cells

The extracts obtained from plasma surface modified membranes did not induce changes in the cell morphology and cell growth in the fibroblasts exposed to these extracts (data not shown), revealing a normal cell morphology and proliferation patterns, similar to those of the negative control, demonstrating the noncytotoxic effect of the membranes modified under different conditions. The results obtained in the MTT test showed, in general, that the leachables prepared from all chitosan membranes surface modified by argon and nitrogen plasma, did not demonstrate any cytotoxic effect. However, the membranes treated with nitrogen plasma exhibited significant differences in terms of cell viability when compared to argon plasma treated membranes. In fact, for the extracts obtained from chitosan membranes surface modified by nitrogen, the percentage of cell viability oscillated between $70 \%$ and $90 \%$ (Figure 1), while the chitosan membranes surface modified by argon showed lower cell viability, in special the formulations ChtP6 (20w20m$\mathrm{Ar}$ ) and ChtP7 (20w30m-Ar), where the percentage obtained varied between $40 \%$ and $45 \%$ (Figure 1). This behavior can be explained according to XPS results, which shows that the argon plasma produced an etching process on the polymer surface for shorter exposition times (10 and $20 \mathrm{~min}$ ), while the nitrogen plasma promoted etching only after an extended treatment [4]. This might result in the formation of some residues accumulated in the groves of the rough 


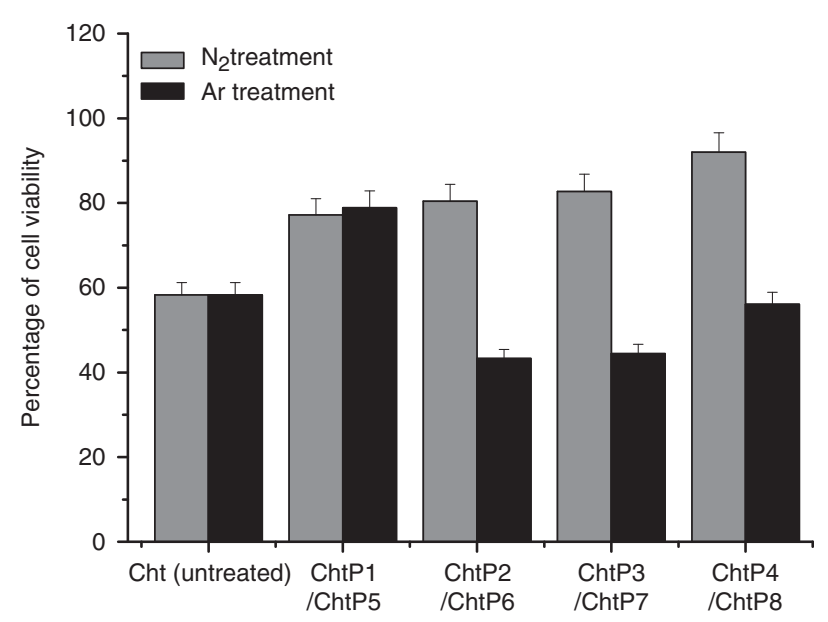

Figure 1. Percentage of cell viability, obtained from the MTT assay, of the fibroblast exposed to extracts of chitosan membranes surface modified by argon and nitrogen plasma (at different conditions).

surface that are released during the extraction and possibly increasing the concentration of leachables. Nevertheless, the cells in contact with extracts obtained from all membranes formulations did not show significant morphological changes when compared with the negative control, demonstrating no signs of any cytotoxicity effect. These results show that these treatments do not affect the noncytotoxic behavior of the chitosan membranes.

\section{Effect of Surface Modification in Chitosan Membranes Using Argon} and Nitrogen Plasma Treatments in Cell Adhesion and Proliferation

Direct contact assay was used to evaluate the cell adhesion and proliferation on chitosan membranes surface modified by argon and nitrogen plasma treatments. In general, the SEM and optical microscopy observations performed after different culture periods, revealed a higher cell adhesion and proliferation of the fibroblast in contact with the chitosan membranes modified with argon and nitrogen plasma as compared to nonmodified membranes (Figures 2 and 3 ).

The cell proliferation in all chitosan membranes modified by both treatments increased with culture time, while for nontreated membranes this was not evident. In fact, surface-treated membrane exhibited the formation of a cell monolayer on the surfaces about $70-80 \%$ confluent after 7 days and $80-100 \%$ confluent after 14 days of culture. 

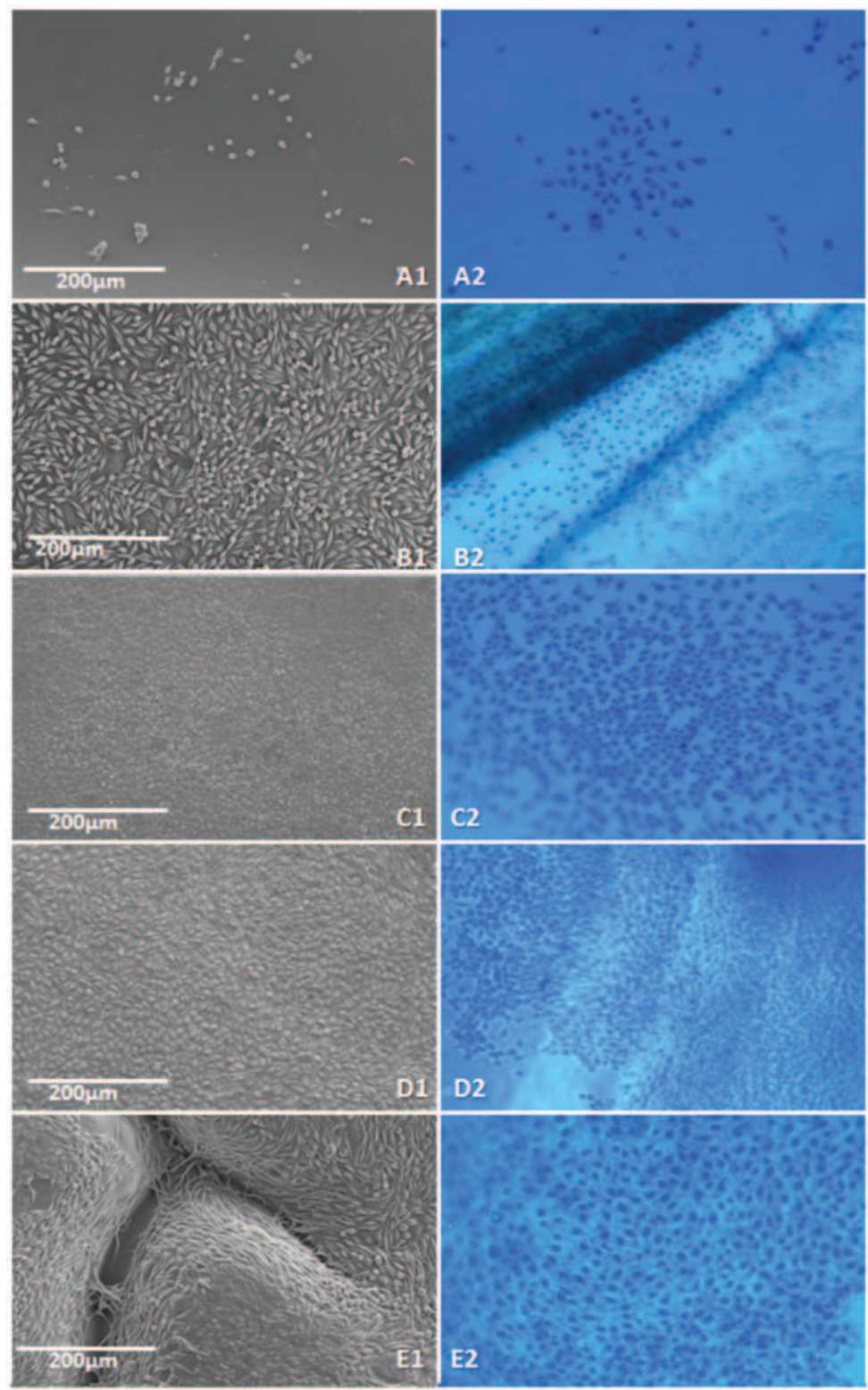

Figure 2. SEM (left) and optical micrographs after blue methylene staining (right $-10 \times$ ) of L929 fibroblasts cultured on untreated chitosan membranes (control) and chitosan membranes modified by nitrogen plasma (using different conditions) after 7 days of culture: A1-A2: Cht (untreated chitosan membranes); B1-B2: ChtP1; C1-C2: ChtP2, D1-D2: ChtP3; E1-E2: ChtP4. 

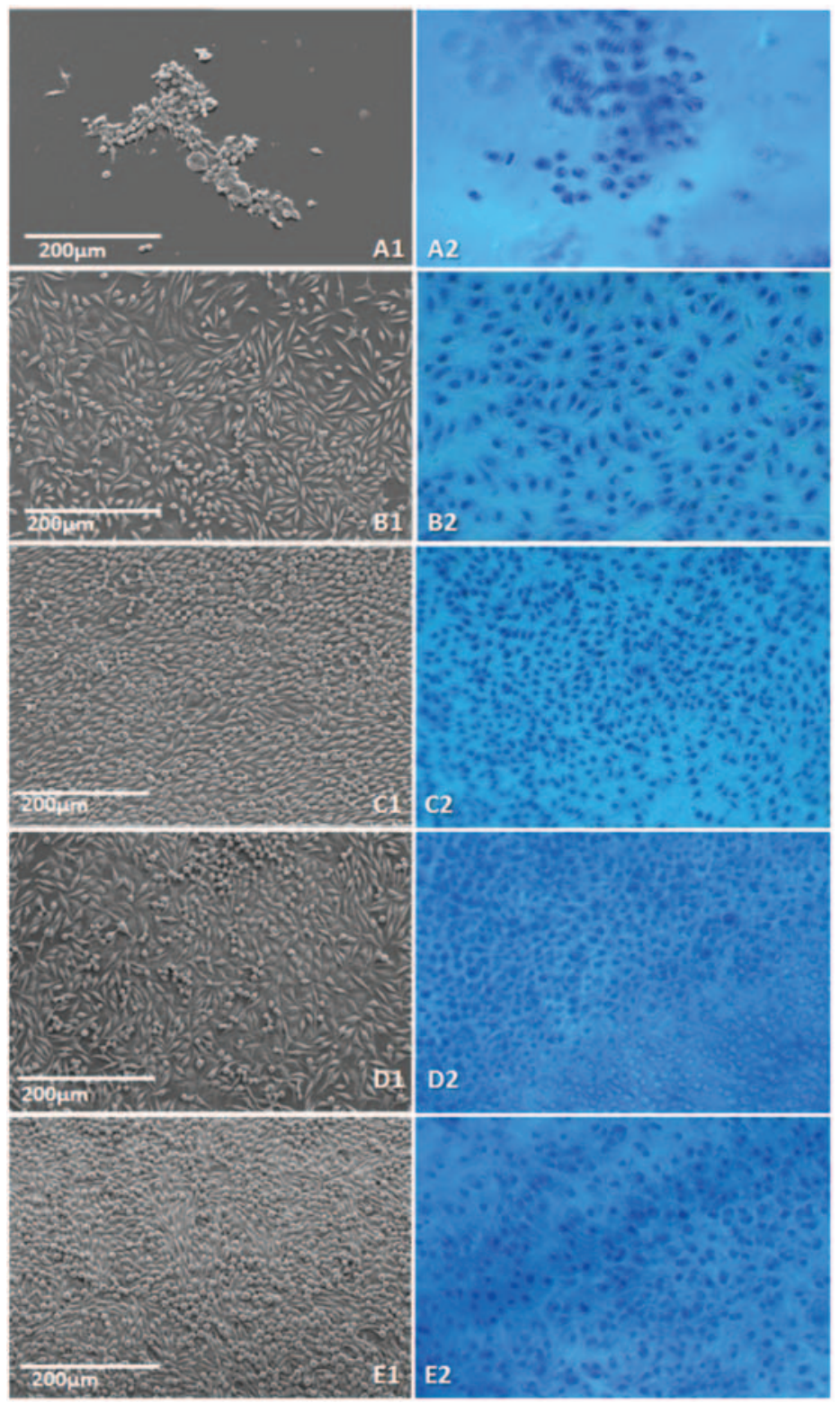

Figure 3. SEM (left) and optical micrographs after blue methylene staining (right $-10 \times$ ) of L929 fibroblasts cultured on untreated chitosan membranes (control) and chitosan membranes modified by argon plasma (using different conditions) after 7 days of culture: A1-A2: Cht (untreated chitosan membranes); B1-B2: ChtP5; C1-C2: ChtP6, D1-D2: ChtP7; E1-E2: ChtP8. 
In the optical and SEM pictures (Figures 2 and 3) it is possible to observe the fibroblasts spreading onto the surface of the membranes exhibiting a flattened morphology that demonstrated a good adherence to the surface. In contrast, in untreated membranes (Cht), the fibroblasts exhibited round morphology, showing low cell adherence and proliferation. Chitosan untreated membrane did not induce neither cell adherence nor proliferation onto the surface with increasing culturing times.

As mentioned before, plasma treatment offers a method of altering the surface characteristics of materials without affecting the material's physical properties $[3,28]$. Previous studies [28,29] showed that the surface functional groups resulting after plasma treatment contained high levels of oxygen and nitrogen; both groups are known to enhance cell adhesion. The literature describes [29] that nitrogen-containing plasma are widely used to improve wettability, printability, biodegradability, and biocompatibility of polymers surfaces. Nitrogen plasma has been used to provide surface amino binding sites for immobilization of heparin on a variety of polymers surfaces [29]. Also, oxygen functionalities are always incorporated in nitrogen-plasma-treated polymer surfaces. The incorporation of oxygen on polymer surfaces after and during nonoxygen plasma treatments is a common phenomenon. Free radicals that are created on a polymer surface can react with oxygen during a plasma treatment. Furthermore, free radicals that remain on a polymer surface after a plasma treatment will react with oxygen when the surface is exposed to the atmosphere [29]. The presence of free radicals and oxygen incorporated by nitrogen plasma in chitosan membranes may explain our results, which demonstrate and enhance cell spread and proliferation after plasma treatments in chitosan membranes.

Figure 3 shows the fibroblasts cells attachment to chitosan membranes modified by argon plasma, where the cellular response was similar to that observed in nitrogen-treated membranes. Argon plasma is known for producing bond breaking and introducing oxygen groups on the surface materials [30]; these groups can be important in the cell attachment and cell growth because the presence of oxygen-containing functional groups such as carboxyl can increase acidity of the surface. Previous studies $[5,28,31]$ using different plasma treatments containing high levels of oxygen and nitrogen groups exhibited similar behavior, that is, enhanced cell adhesion were obtained. In our study, the incorporation of possible oxygen groups and free radicals onto chitosan membranes surface by means of argon and nitrogen plasma enabled high cell proliferation and cell attachment in a short period (a few days) of culture (more details about the surface modifications onto 

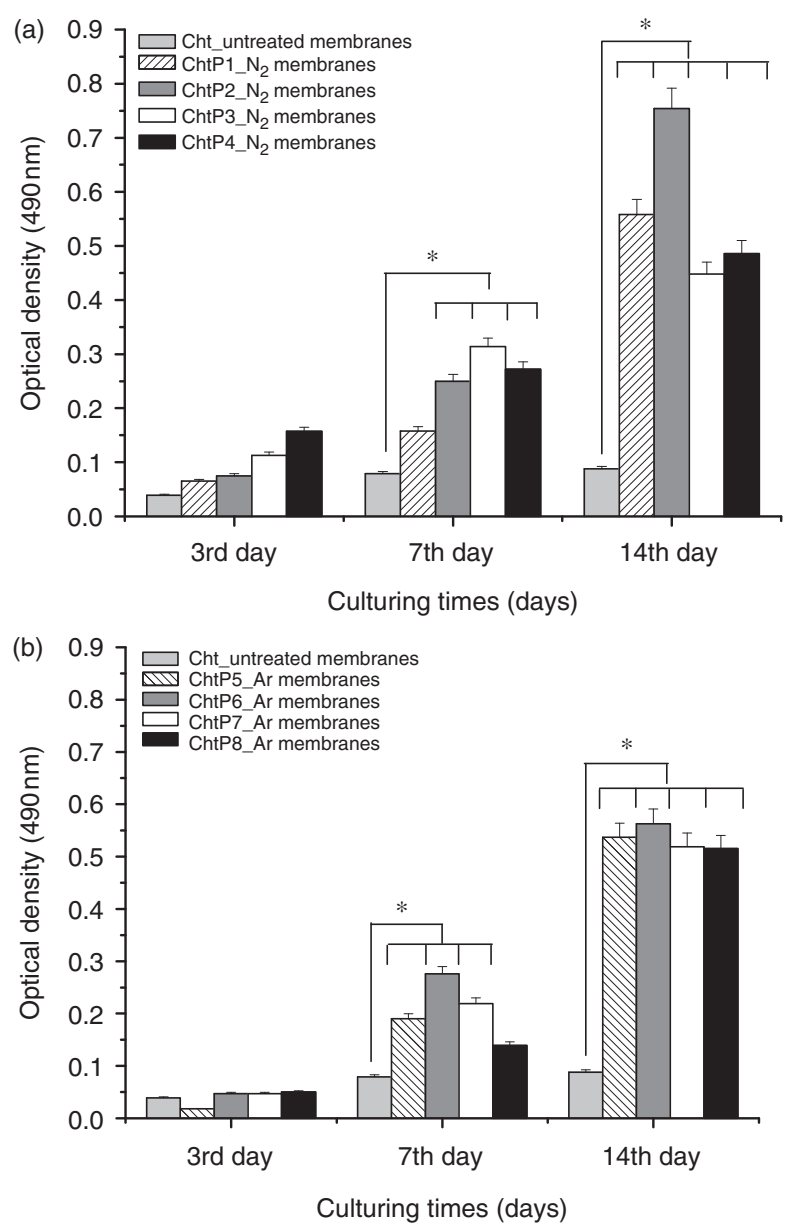

Figure 4. Cell viability and proliferation results obtained using the MTS test. Cell density used was $8 \times 10^{4}$ cells $/ \mathrm{cm}^{2}$. For the control, the same amount of cells was seeded on 24 culture well plates. Cells were kept in culture for 3, 7, and 14 days: (a) Optical density (OD) obtained in chitosan membranes treated by nitrogen plasma, (b) OD obtained in chitosan membranes treated by argon plasma. Asterisk (*) indicates that the cell viability onto chitosan membranes treated by argon and nitrogen plasma was significantly higher $(p<0.05)$ than the untreated chitosan membranes after 7 and 14 days of culture.

plasma-chitosan membranes can be found elsewhere [4]). In contrast, untreated chitosan membranes showed not to be favorable for cell attachment and proliferation like it has been reported in previous studies [32]. This result can be associated to the monopolar basic nature 
of chitosan, which does not interact well with the bipolar extracellular matrix proteins present in the bovine serum proteins of the culture medium [30]. Moreover, the roughness in the chitosan-modified membranes may additionally contribute for a better adhesion of fibroblasts on these surfaces.

The results obtained in the MTS test (Figure 4) suggested that, in general, nitrogen membranes showed a higher cell proliferation as compared with argon treated membranes. Nevertheless, both treatments demonstrated enhanced cell proliferation and viability, as compared to nontreated chitosan membranes, which exhibited low cell proliferation, in accordance with the SEM observations.

The results obtained also demonstrated that the plasma conditions corresponding to ChtP2 and ChtP6 produced the best cell proliferation for all the culture periods studied. These results indicate that low power and short time conditions of exposure to nitrogen or argon plasma is enough to produce improvement in the cell growth on chitosan-based membranes, since it was possible to observe cells attaching and spreading on the chitosan surface membrane after 3 days of culture.

\section{CONCLUSIONS}

The surface modification of chitosan membranes with nitrogen and argon plasma were found to promote the proliferation of L929 fibroblasts compared to untreated chitosan membrane, where no cell adherence was observed. Cytotoxic assays showed that none of the treatments (under all studied conditions) induced any cytotoxic effect. SEM and optical microscope observations showed a clear enhancement of cell attachment and proliferation using argon and nitrogen plasma modified membranes as compared to untreated chitosan membranes. In fact, after short culturing periods, argon and nitrogen plasma treatment seems to produce better results in terms of cells attachment and proliferation. The data obtained from the MTS test confirmed those results, where again, it was demonstrated that nitrogen and argon plasma treatment performed under optimized conditions, seems to be a suitable technique for modifying surface properties, enhancing the biological response of chitosan membranes. High levels of oxygen and nitrogen obtained after plasma treatment raise the rates of cell proliferation in comparison with untreated membranes. Overall, the results showed that surface modification by plasma to be useful technique to treat chitosan-based material leading to enhanced cell adhesion and proliferation. These findings may be relevant in the development of chitosan-based biomaterials, including in tissue engineering applications. 


\section{ACKNOWLEDGMENTS}

S. M. Luna acknowledges the support of the Programme Alßan - The European Union Programme of High Level Scholarships for Latin America (scholarship $\mathrm{N}^{\circ}$ E04M041362CO) and Silva SS acknowledge the support of the Portuguese Foundation for Science and Technology, (SFRH/BPD/45307/2008). This work was also partially supported by the European Union-funded - STREP project HIPPOCRATES (NMP3-CT2003-505758) and was carried out under the scope of European NoE EXPERTISSUES (NMP3-CT-2004-500283).

\section{REFERENCES}

1. Gumpenberg, T., Heitz, J., Bauerle, D. et al. Adhesion and Proliferation of Human Endothelial Cells on Photochemically Modified Polytetrafluoroethylene, Biomaterials, 2003: 24: 5139-5144.

2. Yang, F., Li, X., Cheng, M., Gong, Y., Zhao, N. and Zhang, X. Performance Modification of Chitosan Membranes Induced by Gamma Irradiation, J. Biomater. Appl., 2002: 16: 215-226.

3. Oehr, C. Plasma Surface Modification of Polymers for Biomedical Use, Nucl. Instr. Meth. Phys. Res. B, 2003: 208: 40-47.

4. Silva, S.S., Luna, S.M., Gomes, M.E. et al. Plasma Surface Modification of Chitosan Membranes: Characterization and Preliminary Cell Response Studies, Macromol. Biosci., 2008: 8: 568-576.

5. Zhu, X., Chian, K., Chan-Park, M. and Lee, S. Effect of Argon-Plasma Treatment on Proliferation of Human-skin Derived Fibroblast on Chitosan Membrane In Vitro, J. Biomed. Mater. Res. Part A, 2005: 73A: 264-274.

6. Pashkuleva, I., Marques, A.P., Vaz, F. and Reis, R.L. Surface Modification of Starch Based Blends Using Potassium Permanganate-Nitric Acid System and Its Effect on the Adhesion and Proliferation of Osteoblast-like Cells, J. Mater. Sci.: Mater. Med., 2005: 16: 81-92.

7. Pérez, P., Marques, A., Silva, R., Pashkuleva, I. and Reis, R. Effect of Chitosan Surface Modification Via Plasma Induced Polymerization on the Adhesion of Osteoblast-like Cells, J. Mater. Chem., 2007: 17: 4064-4071.

8. Ratner, B. Surface Modification of Polymers: Chemical, Biological and Surface Analytical Challenges, Biosens. Bioelectron., 1995: 10: 797-804.

9. Kumar, M., Muzzarelli, R., Muzzarelli, C., Sashiwa, H. and Domb, A. Chitosan Chemistry and Pharmaceutical Perspectives, Chem. Rev., 2004: 104: $6017-6084$.

10. Martino, A.D., Sittinger, M. and Risbud, M.V. Chitosan: A Versatile Biopolymer for Orthopaedic Tissue-engineering, Biomaterials, 2005: 26: 5983-5990.

11. Mi, F.-L., Shyu, S.-S., Wu, Y.-B., Lee, S.-T., Shyong, J.-Y. and Huang, R.-N. Fabrication and Characterization of a Sponge-like Asymmetric Chitosan Membrane as a Wound Dressing, Biomaterials, 2001: 22: 165-173. 
12. Mi, F.-L., Wu, Y.-B., Shyu, S.-S., Chao, A.-C., Lai, J.-Y. and Su, C.-C. Asymmetric Chitosan Membranes Prepared by Dry/Wet Phase Separation: A New Type of Wound Dressing for Controlled Antibacterial Release, J. Membr. Sci., 2003: 212: 237-254.

13. Ueno, H., Yamada, H., Tanaka, I. et al. Accelerating Effects of Chitosan for Healing at Early Phase of Experimental Open Wound in Dogs, Biomaterials, 1999: 20: 1407-1414.

14. Ueno, H., Mori, T. and Fujinaga, T. Topical Formulations and Wound Healing Applications of Chitosan, Adv. Drug Deliv. Rev., 2001: 52: 105-115.

15. Castner, D.G. and Ratner, B.D. Biomedical Surface Science: Foundations to Frontiers, Surf. Sci., 2002: 500: 28-60.

16. Kumar, M.N.V.R., Muzzarelli, R.A.A., Muzzarelli, C., Sashiwa, H. and Domb, A.J. Chitosan Chemistry and Pharmaceutical Perspectives, Chem. Rev., 2004: 104: 6017-6084.

17. Zielinski, B.A. and Aebischer, P. Chitosan as a Matrix for Mammalian Cell Encapsulation, Biomaterials, 1994: 15: 1049-1056.

18. Shi, C., Zhu, Y., Ran, X., Wang, M., Su, Y. and Cheng, T. Therapeutic Potential of Chitosan and Its Derivatives in Regenerative Medicine, J. Surg. Res., 2006: 133: 185-192.

19. Mori, T., Okumura, M., Matsuura, M. et al. Effects of Chitin and Its Derivatives on the Proliferation and Cytokine Production of Fibroblasts In Vitro, Biomaterials, 1997: 18: 947-951.

20. 10993-5, ISO/EN Biological Evaluation of Medical Devices - Part 5 Test for Cytotoxicity, In Vitro Methods: 8.2 Test on Extracts.

21. Kirkpatrick, C.J. and Dekker, A. (1992). In Biomaterials - Tissue Interfaces, 1st edn, Amsterdam, Elsevier Science Publishers.

22. Gomes, M.E., Reis, R.L., Cunha, A.M., Blitterswijk, C.A. and de Bruijn, J.D. Cytocompatibility and Response of Osteoblastic-like Cells to Starch-based Polymers: Effect of Several Additives and Processing Conditions, Biomaterials, 2001: 22: 1911-1917.

23. Silva, S.S., Santos, M.I., Coutinho, O.P., Mano, J.F. and Reis, R.L. Physical Properties and Biocompatibility of Chitosan/Soy Blended Membranes, J. Mater. Sci.: Mater. Med., 2005: 16: 575-579.

24. Salgado, A.J., Gomes, M.E., Chou, A., Coutinho, O.P., Reis, R.L. and Hutmacher, D.W. Preliminary Study on the Adhesion and Proliferation of Human Osteoblasts on Starch-based Scaffolds, Mater. Sci. Eng., C, 2002: 20: 27-33.

25. Salgado, A.J., Coutinho, O.P. and Reis, R.L. Novel Starch-based Scaffolds for Bone Tissues Engineering Cytotoxicity, Cell Culture and Protein Expression, Tissue Eng., 2004: 10: 465-474.

26. Chu, P., Chen, J., Wang, L. and Huang, N. Plasma-surface Modification of Biomaterials, Mater. Sci. Eng. Rev., 2002: 36: 143-206.

27. Gerenser, L.J., Grace, J.M., Apai, G. and Thompson, P. Surface Chemistry of Nitrogen Plasma-treated Poly(ethylene-2,6-naphthalate): XPS, HREELS and Static SIMS Analysis, Surf. Interf. Anal., 2000: 29: 12.

28. Seal, B., Otero, T. and Panitch, A. Review Polymeric Biomaterials for Tissue and Organ Regeneration, Mater. Sci. Eng. Rev., 2001: 34: 147-230. 
29. Chan, C.-M., Ko, T.-M. and Hiraoka, H. Polymer Surface Modification by Plasmas and Photons, Surf. Sci. Rep., 1996: 24: 1-54.

30. Zhu, X., Chian, K.S., Chan-Park, M.B.E. and Lee, S.T. Effect of Argon-Plasma Treatment on Proliferation of Human-skin-derived Fibroblast on Chitosan Membrane In Vitro, J. Biomed. Mater. Res., 2005: 73A: 264-274.

31. Ohl, A. and Schröder, K. Plasma-induced Chemical Micropatterning for Cell Culturing Applications: A Brief Review, Surf. Coatings Technol., 1999: 116-119: 820-830.

32. Chatelet, C., Damour, O. and Domard, A. Influence of the Degree of Acetylation on Some Biological Properties of Chitosan Films, Biomaterials, 2001: 22: 261-268. 